\title{
Decentralized Optimization for a Novel Control Structure of HVAC System
}

\author{
Shiqiang Wang, ${ }^{1,2}$ Jianchun Xing, ${ }^{1}$ Ziyan Jiang, ${ }^{2}$ and Juelong $\mathrm{Li}^{1}$ \\ ${ }^{1}$ College of Defense Engineering, PLA University of Science and Technology, Nanjing, Jiangsu 210007, China \\ ${ }^{2}$ Building Energy Research Center, Tsinghua University, Beijing 100084, China \\ Correspondence should be addressed to Shiqiang Wang; swiptwsq@163.com
}

Received 23 November 2015; Revised 27 February 2016; Accepted 3 April 2016

Academic Editor: George S. Dulikravich

Copyright (c) 2016 Shiqiang Wang et al. This is an open access article distributed under the Creative Commons Attribution License, which permits unrestricted use, distribution, and reproduction in any medium, provided the original work is properly cited.

\begin{abstract}
A decentralized control structure is introduced into the heating, ventilation, and air conditioning (HVAC) system to solve the high maintenance and labor cost problem in actual engineering. Based on this new control system, a decentralized optimization method is presented for sensor fault repair and optimal group control of HVAC equipment. Convergence property of the novel method is theoretically analyzed considering both convex and nonconvex systems with constraints. In this decentralized control system, traditional device is fitted with a control chip such that it becomes a smart device. The smart device can communicate and operate collaboratively with the other devices to accomplish some designated tasks. The effectiveness of the presented method is verified by simulations and hardware tests.
\end{abstract}

\section{Introduction}

The heating, ventilation, and air conditioning (HVAC) system is responsible for providing a high-quality and healthy environment for the building's occupants. It comprises a set of interconnected subsystems, including the heating and cooling plants, the ventilation system, and one or more zones served by the terminal units of the ventilation system. The control system is an indispensable part of HVAC system. An HVAC control system consists of several hardware components, such as sensors, electrical and mechanical actuators, and controllers, and is highly complex and nonlinear, especially in the case of large-scale buildings [1].

The current DDC based control architecture in HVAC systems has several deficiencies during construction and operation. The construction problem is the high labor cost for the establishment of the basic network. In some cases, this task is even unfeasible and can be exceedingly demanding. During practical operations, the traditional control system needs information transmission to the supervising computer for control and alarm processes, which can cause severe link congestion and operational lag. The implementation of DDC based control system also encounters difficulties when the system scale changes. What is more, the entire system cannot continue to process information and always develops chain breaks if the supervising center breaks down. In addition, the information security of hierarchical control structure is faced with challenge as well. Most HVAC control systems today are not fully operational and are trivial for the reasons mentioned above.

Under the conventional HVAC control structure, the majority of sensor fault detection and diagnosis (SFDD) methods are based on a centralized algorithm [2-19]. To the best of our knowledge, these methods have a series of disadvantages, such as poor adaptability and instantaneity. Therefore, few SFDD methods for HVAC systems have been applied in practice. Sensor fault repair is considered a key problem due to the increasing number of sensors for the monitoring and control of HVAC systems.

For the optimal control of devices group, including cooling towers, chillers, and pumps, the current control structure needs considerable configuration and can hardly reach optimal operation. The reason is that the accurate model of the total HVAC system is complex and is yet to be established in some sense. The group control in large-scale HVAC control systems is another challenge encountered [20]. 


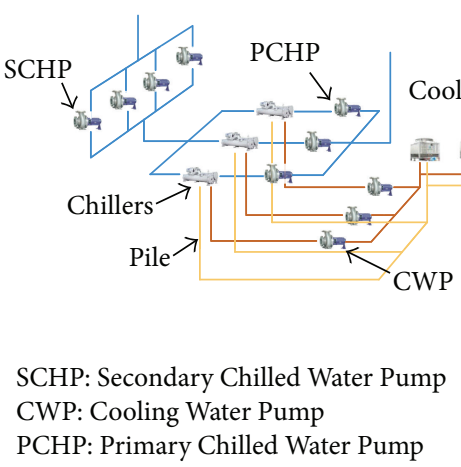

(a)

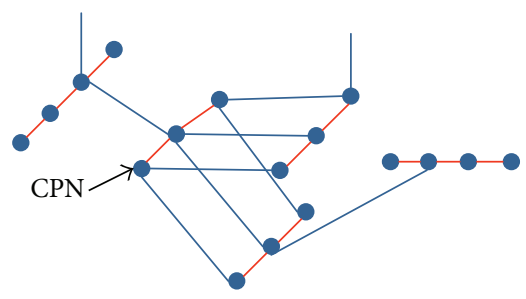

(b)

FIgURE 1: HVAC control systems. (a) Typical HVAC system. (b) Decentralized HVAC control system.

As the conventional control structure is a barrier for the broad application of HVAC control systems, new architecture with features such as easy extension, convenient implementation, and plug-and-play operation has never been more desirable. To meet practical needs and theoretical challenges, a decentralized HVAC control system is proposed [21]. The objective and main contribution of the present study are the design and analysis of a novel decentralized method for the SFDD and the group control based on a decentralized HVAC control system.

\section{Decentralized HVAC Control System}

The HVAC system is a large-scale interconnected nonlinear system as shown in Figure 1(a). Thus, the DDC based control approach may be less suitable compared with a decentralized approach. The decentralized control system consists of several autonomous and network-connected computing nodes (CPNs), in which a microcontroller unit is embedded. According to the vision of a decentralized method, traditional devices can be upgraded and transformed into smart devices through the incorporation of the CPNs.

For simplicity, the topology is used to denote regional relationships: each subsystem is represented by a node and the edges refer to physical connectivity as depicted in Figure 1(b). Notably, the effect of a physical field (e.g., humidity and temperature) in most HVAC control systems is gradual and mainly reflected in adjacent areas. Therefore, each CPN (agent) can only communicate with its immediate neighboring agents, which are connected through network cables. Any computing task must be accomplished in a decentralized and coordinated manner by the CPNs. The decentralized HVAC control system simplifies the configuration and facilitates the installation. In this case, complicated onsite modeling, configuration, commissioning, and other developing work are simplified to the wiring of communication connection among smart devices. Thus, this novel flat control structure is flexible and plug-and-play. The present study designs a novel decentralized mechanism assisted by a decentralized optimization method.

\section{Decentralized Optimization}

Decentralized approaches remove the requirement of central coordination and enable individual devices to determine their own actions based on local and neighboring information.

3.1. Problem Statement. Most research and applications can be abstracted as constrained optimization problems. The minimum optimization is considered in this study. Thus, the centralized optimization can be stated as

$$
\begin{array}{cl}
\min _{\left\{\mathbf{x} \in \mathbf{R}^{\mathbf{n}}\right\}} & \sum_{i=1}^{N} f_{i}\left(\mathbf{x}_{i}\right) \\
\text { s.t. } & g(\mathbf{x}) \leq 0 \\
& h(\mathbf{x})=0,
\end{array}
$$

where the variable $\mathbf{x}_{i}, i=1, \ldots, N$, with coupled variables denotes the decision variable of agent $i$ and $N$ is the quantity of agents. The local objective function $f_{i}\left(\mathbf{x}_{i}\right), i=1, \ldots, N$, represents the individual cost of agent $i$ in the actual engineering. Constraints $g(\mathbf{x})$ and $h(\mathbf{x})$ can include the basic physical laws, such as energy, mass, and momentum conservation equations.

The implementation of the centralized optimization problem (1) requires a central processor with access to the total system information. However, the decentralized method focuses on each local agent with restricted information on the whole system. Each agent can exchange information only with its neighboring nodes.

To provide a clear expression, the neighborhood of a given agent $i$ is defined as $N_{i}=\{j \mid i$ th agent has a constraint involving the $j$ th agent $\}$. The set $\mathbf{x}_{i j}, j \in N_{i}$, is introduced to denote the coupled variable, which is the decision variable shared by agents $i$ and $j$. The decentralized structure requires each agent to solve local optimization exclusively based on the information on other agents in its neighborhood. The approach of the present study is based on variable splitting method [22] to obtain an equivalent constrained optimization formulation, which is then addressed in a decentralized 


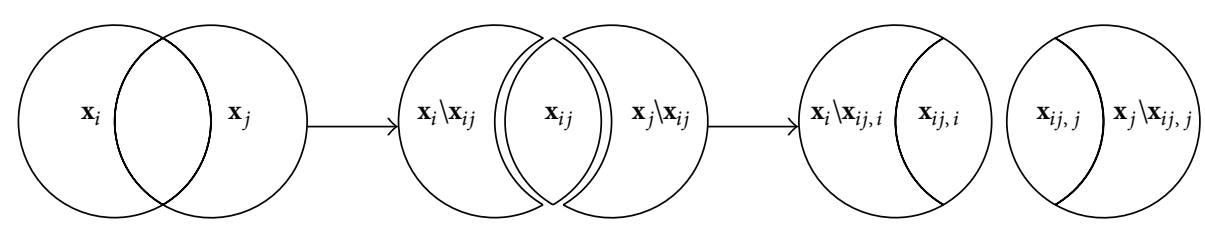

FIGURE 2: Variable splitting method.

manner. The variable splitting method is shown in Figure 2. In $\mathbf{x}_{i}$ and $\mathbf{x}_{j}$, the common variable set $\mathbf{x}_{i j}$ is replaced by $\mathbf{x}_{i j, i}$ and $\mathbf{x}_{i j, j}$, respectively. Moreover, $\mathbf{x}_{i j, i}=\mathbf{x}_{i j, j}$.

Then problem (1) can be transformed into the decentralized optimization problem (2) based on the variable splitting method:

$$
\begin{array}{ll}
\min _{\left\{x_{i}\right\}} & f_{i}\left(x_{i}\right) \\
\text { s.t. } & g_{i}\left(x_{i} \mid x_{i j, i}\right) \leq 0 \\
& h_{i}\left(x_{i} \mid x_{i j, i}\right)=0 \\
& x_{i j, j}=x_{i j, i}, \quad j \in N_{i},
\end{array}
$$

where the notation $g\left(\cdot \mid x_{i j, i}\right), h\left(\cdot \mid x_{i j, i}\right)$ represents the function of $x_{i}$ in which the neighborhood state $x_{i j}$ is fixed. Node $i$ can solve problem (2) after it determines the neighborhood value $x_{i j, j}$ provided that $x_{i j}$ is received from its neighbors in the previous iteration. If each objective function is as close as possible to its minimum, then the optimal solution of the centralized optimization problem can be obtained while also ensuring that the coupled constraints are not violated across the nodes. Consequently, each node can realize a decentralized and autonomous operation. For the decentralized optimization problem, optimality is defined using the Nash equilibrium [23]. The expression $x^{* \mathrm{de}}=$ $\left[x_{1}{ }^{* \mathrm{de}}, \ldots, x_{i}{ }^{* \mathrm{de}}, \ldots, x_{N}{ }^{* \mathrm{de}}\right]$ is a Nash equilibrium for the optimization problem if

$$
f_{i}\left(x_{i}^{* \mathrm{de}}\right) \leq f_{i}\left(x_{i}\right), \quad i=1,2, \ldots, N
$$

where $N$ is the quantity of agents. Assuming that the optimization problems were convex and differential, Inalhan gave the decentralized algorithm and the corresponding equivalence of the centralized optimization and decentralized optimization [23].

However, most optimization problems in engineering are usually nonderivable, discontinuous, constrained, and highly nonlinear problems with numerous local optima. Thus, the present study proposes an iterative method to solve the decentralized optimization of HVAC control system.

3.2. Algorithm Design. The decentralized estimation distribution algorithm (EDA) is provided to solve the decentralized optimization problem. EDA is an evolutionary algorithm based on probability distribution [24]. The algorithm is adapted in a decentralized manner: each agent executes identical evolutionary algorithms with neighboring coordination. Moreover, the penalty method is applied to solve the consensus optimization. For the decentralized optimization model presented in problem (2), the modified penalty cost function of the $i$ th agent is considered as

$$
\begin{array}{ll}
\min _{\left\{x_{i}\right\}} & f_{i}\left(x_{i}\right)+\sum_{j \in N_{i}} u_{i}\left\|x_{i j, j}-x_{i j, i}\right\|^{2} \\
\text { s.t. } & g_{i}\left(x_{i} \mid x_{i j, i}\right) \leq 0 \\
& h_{i}\left(x_{i} \mid x_{i j, i}\right)=0
\end{array}
$$

where the penalty factor $u_{i}>0$, the initial value of which is finite, approaches infinity when the iteration progresses. When $u_{i}$ is small, or in an extreme case $u_{i}=0$, the optimal solution minimizes agent $i$ without considering the agent $j$, that is, without considering the penalty terms. In other words, during the penalty function transformation, $x_{i j, i}$ and $x_{i j, j}$ do not need to be equal when $u_{i}$ is not large enough. When $u_{i}$ increases, the optimal solution considers reducing the penalty terms. If $u_{i}$ approaches infinity, then $x_{i j, i}=x_{i j, j}$.

The analysis cited in the preceding paragraph makes analyzing the convergence of the presented algorithm possible. In short, the $i$ th agent collects the value of the coupled variables from the neighborhood $N_{i}$ and optimizes with the local $u_{i}$. To present a clear expression, the key algorithm is described in the flow chart in Figure 3.

The presented decentralized algorithm can be treated as a synchronous iteration. In the form of synchronous iteration, all agents are initially optimized on the basis of the desired solutions. The solution set is then passed to its neighborhood and is reoptimized for each solution set received at each step.

3.3. Convergence Analysis. It is proved that the decentralized optimization algorithm converges to the centralized optimality in this section.

For the discrete EDA, the solution space in each local agent $i, i=1, \ldots, N$, is defined as $\Omega=\Omega_{1} \cap \Omega_{2} \cap \cdots \cap \Omega_{n}$, where $\Omega_{s}, s=1,2, \ldots, n$, is the space of the $s$ th variable and $n$ is the length of chromosome vector. For the sth variable, $\Omega_{s}=\left\{x_{1}, x_{2}, \ldots, x_{r s}\right\}$, where $r_{s}$ is the quantity of the value states, and $|\Omega|=r_{1} \cdot r_{2} \cdot \ldots \cdot r_{n}$. If $D_{l}$ is the $l$ th generation that contains $M$ chromosomes, then $D_{l}$ is written as $D_{l}=$ $\left(d_{1 l}, d_{2 l}, \ldots, d_{M l}\right)$, where $d_{i l}$ denotes the $i$ th chromosome among $D_{l}$.

For all $x \in \Omega$, a chromosome $x^{*}$ satisfies $f_{i}\left(x^{*}\right) \leq$ $f_{i}(x)(i=1, \ldots, N)$ and is contained in generation $D^{*}$. Considering that the $l$ th generation depends only on the $(l-1)$ th generation, Markov models are applied to describe 


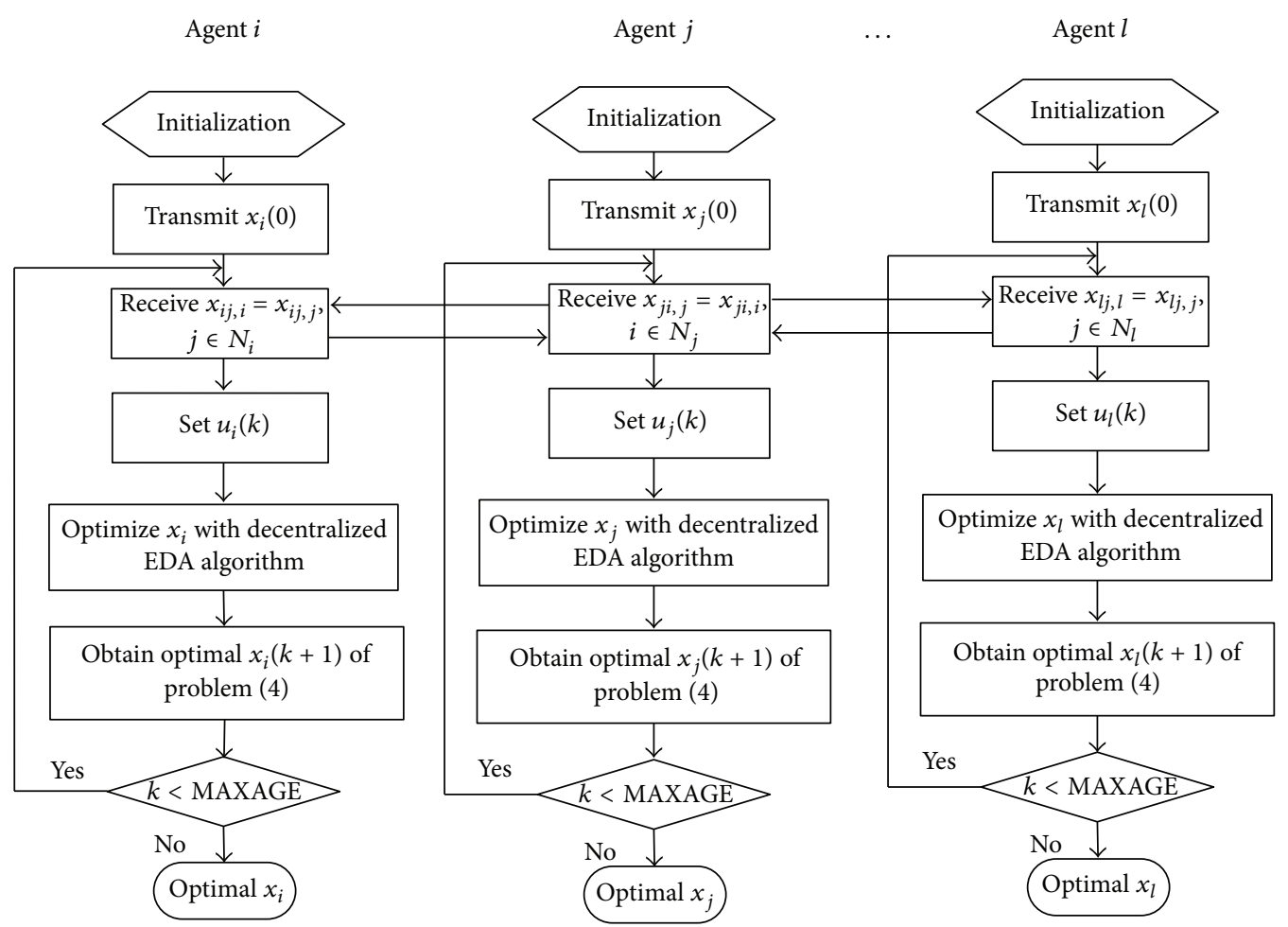

FIGURE 3: Flow chart of the decentralized optimization algorithm.

this evolutionary process. For a generation, the transfer probability of the value state is

$$
p_{i j}(t)= \begin{cases}G_{i j}(t), & \forall j \neq i, \\ 1-\sum_{|\Omega|} G_{i j}(t), & j=i,\end{cases}
$$

where $G_{i j}(t)$ is the transfer probability from states $i$ to $j$. It can be proved that the EDA can reach generation $D^{*}$ and thus converge to a local optimization (or global optimization for each agent $i$ ) with a probability of 1 [24].

For the continuous case, an elite-preserving operator is used in the presented algorithm. This operator favors the $g$ elites of a population by giving them an opportunity to be directly carried over to the next generation. Thus, the new population consists of $(M-g)$ new chromosomes, with $g<$ $M$. The following theorems of probability and statistics are introduced to complete the proof process.

Theorem 1. For any $\varepsilon>0$, if

$$
P\left\{\bigcap_{n=1}^{\infty} \bigcup_{k \geq n}\left[\left|\xi_{n}-\xi\right| \geq \varepsilon\right]\right\}=0
$$

then the random $\left\{\xi_{n}\right\}$ exists almost everywhere and converges to $\xi$ with a probability of 1 .
Theorem 2. The present study assumes that $A_{1}, A_{2}, \ldots$, is the event sequence based on probability and defines $p_{k}=P\left\{A_{k}\right\}$. If

$$
\sum_{k=1}^{\infty} p_{k}<\infty
$$

then

$$
P\left\{\bigcap_{n=1}^{\infty} \bigcup_{k \geq n} A_{k}\right\}=0
$$

If

$$
\sum_{k=1}^{\infty} p_{k}=\infty
$$

and $A_{k}$ are independent of one another, then

$$
P\left\{\bigcap_{n=1}^{\infty} \bigcup_{k \geq n} A_{k}\right\}=1 .
$$

The present study assumes that the search space $\Omega$ of problem (4) is a closed and bounded region. The objective function is taken as the fitness function $J(x)$, and the optimal solution set is expressed as

$$
D_{0}=\left\{x \in \Omega|| J(x)-J^{*} \mid<\varepsilon\right\},
$$

where $J^{*}=\min \{J(x): x \in \Omega\}$. The $M$ chromosomes of EDA can be divided into the following properties: 
(I) At least one chromosome belongs to $D_{0}$, which is written as $S_{0}$.

(II) All $M$ chromosomes belong to $D_{1}$, which is written as $S_{1}$, where

$$
D_{1}=\Omega \backslash D_{0}
$$

If $q_{i j}(i, j=0,1)$ represents the probability that the $(k+1)$ th generation $x(k+1)$ transfers to $S_{j}$ when the $k$ th generation $x(k)$ keeps the state of $S_{i}$, then the following conditions hold:

(I) $x(k)$ at $S_{0}$ and $q_{00}=1$.

(II) $x(k)$ at $S_{1}$ and $q_{11} \leq c$, where $c \in(0,1)$.

Proof. Conditions (I)-(II) are proved in sequence as follows:

(I) This algorithm uses the elite-preserving operator. Thus, situation (I) is obviously valid.

(II) For any $\varepsilon>0$, the present study assumes that $x_{0} \in$ $\left\{x \in \Omega|| J(x)-J^{*} \mid<\varepsilon / 2\right\}$. Thus, $r>0$ exists satisfying

$$
\left|J(x)-J\left(x_{0}\right)\right|<\frac{\varepsilon}{2},
$$

when

$$
\left\|x-x_{0}\right\|_{\infty}=\max _{1 \leq i \leq l}\left|x^{i}-x_{0}{ }^{i}\right| \leq r
$$

where $l$ denotes the length of the chromosome.

Define

$$
Q_{x_{0}, r}=\left\{x \in \Omega \mid\left\|x-x_{0}\right\|_{\infty} \leq r\right\},
$$

so that

$$
Q_{x_{0}, r} \subset D_{0}
$$

due to

$$
\begin{aligned}
\left|J(x)-J^{*}\right| & \leq\left|J(x)-J\left(x_{0}\right)\right|+\left|J\left(x_{0}\right)-J^{*}\right|<\frac{\varepsilon}{2}+\frac{\varepsilon}{2} \\
& =\varepsilon .
\end{aligned}
$$

The probability that $x$ transfers to $S_{0}$ can be defined by

$$
P\left\{x_{+} \in Q_{x_{0}, r}\right\}=\prod_{i=1}^{l} P\left\{\left|x_{+}{ }^{i}-x_{0}{ }^{i}\right| \leq r\right\},
$$

where $x_{+}$is the chromosome of a new generation, $x_{+}{ }^{i}$ and $x_{0}{ }^{i}$ are the $i$ th elements of $x_{+}$and $x_{0}$, respectively, and $i=$ $1,2, \ldots, l$. Given that the evolutionary computation of EDA is based on probability, each element of the chromosome generates a series of the probability function $g\left(x^{i}\right)$, with $i=$ $1,2, \ldots, l$.

For $x_{+}{ }^{i}$,

$$
x_{+}{ }^{i} \sim g\left(x^{i}\right)
$$

and thus

$$
P\left\{x_{+} \in Q_{x_{0}, r}\right\}=\prod_{i=1}^{l} \int_{x_{0}{ }^{i}-r}^{x_{0}{ }^{i}+r} g\left(x^{i}\right) d x^{i} .
$$

For simplicity, $P\left\{x_{+} \in Q_{x 0, r}\right\}$ can be denoted by

$$
P_{1}\left(x_{+}\right)=P\left\{x_{+} \in Q_{x 0, r}\right\}
$$

and then clearly

$$
0<P_{1}\left(x_{+}\right)<1 \text {. }
$$

Define

$$
P_{1}\left(y_{0}\right)=\min P_{1}\left(x_{+}\right)
$$

so that

$$
P_{1}\left(y_{0}\right) \leq P_{1}\left(x_{+}\right) \leq q_{10} .
$$

As

$$
q_{11}+q_{10}=1
$$

the following result can be achieved:

$$
q_{11}=1-q_{10} \leq c(0<c<1),
$$

where the constant $c$ is

$$
c=1-P_{1}\left(y_{0}\right)
$$

Proposition 3. The present study assumes that $x_{j}(m), j=$ $1,2, \ldots, M$, is the population of the mth generation of EDA, which consists of $M$ chromosomes. If the fitness function $J(x)$ is continuous on the bounded region $\Omega$, the optimal solution $x^{*}(m)$ converges to the local optimization for agent $i$ with a probability of 1 , and

$$
x^{*}(m)=\arg \min _{1 \leq j \leq M} J\left(x_{j}(m)\right) .
$$

Proof. For any $\varepsilon>0$, set

$$
\begin{aligned}
& p_{k}=P\left\{\left|J\left(x^{*}(k)\right)-J^{*}\right| \geq \varepsilon\right\}, \\
& p_{k}= \begin{cases}0, & \exists T \in\{0,1,2, \ldots, k\}, x^{*}(T) \in D_{0}, \\
\overline{p_{k}}, & x^{*}(m) \notin D_{0}, m=0,1,2, \ldots, k,\end{cases}
\end{aligned}
$$

and thus, the following equation is obtained:

$$
\overline{p_{k}}=P\left\{x^{*}(m) \notin D_{0}, m=0,1,2, \ldots, k\right\}=q_{11}{ }^{k} \leq c^{k} \text {, }
$$

so that

$$
\sum_{k=1}^{\infty} \overline{p_{k}} \leq \sum_{k=1}^{\infty} c^{k}=\frac{c}{1-c}<\infty .
$$




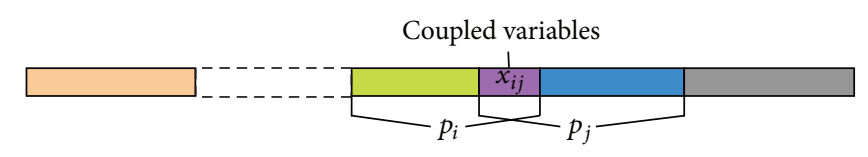

FIGURE 4: Global solution chromosome.

According to Theorem 2,

$$
P\left\{\bigcap_{m=1}^{\infty} \bigcup_{k \geq m}\left[\left|J\left(x^{*}(k)\right)-J^{*}\right| \geq \varepsilon\right]\right\}=0
$$

is true. Therefore, the local solution converges to the local optimization for agent $i$ with a probability of 1 . Proposition 3 is true for each agent in the decentralized control system. Then the validity of the global optimization is deduced in the subsequent paragraphs.

Proposition 4. For the decentralized optimization, $x^{\text {de }}$ converges to the Nash equilibrium of the centralized optimization problem with a probability of 1 .

Proof. According to Proposition 3, the local solution $x_{i}{ }^{\text {de }}$ can converge to the local optimization $x_{i}{ }^{*}$ de for agent $i$ with a probability of 1 . Define the probability as $p_{i}$; that is,

$$
\begin{aligned}
x_{i}{ }^{\text {de }} \stackrel{p_{i}}{\longrightarrow} x_{i}^{* \text { de }}, \\
p_{i}=P\left\{\bigcap_{m=1}^{\infty} \bigcup_{k \geq m}\left[\left|J\left(x_{i}^{\text {de }}(k)\right)-J^{*}\right|<\varepsilon\right]\right\}=1 .
\end{aligned}
$$

According to the limit operation, the global probability can be described as

$$
P=\prod_{i=1}^{N} p_{i}=1 .
$$

For the coupled variables, a similar result is also obtained:

$$
P=\frac{\prod_{i=1}^{N} p_{i}}{p_{i j}}=1
$$

as shown in Figure 4.

Then the present study directly obtains

$$
x^{\mathrm{de}} \stackrel{P}{\longrightarrow} x^{* \mathrm{de}},
$$

where $x^{* \mathrm{de}}=\left[x_{1}{ }^{* \mathrm{de}}, \ldots, x_{i}^{* \mathrm{de}}, \ldots, x_{N}{ }^{* \mathrm{de}}\right]$ is a Nash equilibrium of problem (1). In conclusion, $x^{\text {de }}$ converges to an optimal solution of the centralized optimization problem with a probability of 1 as the iteration step $m \rightarrow \infty$.

3.4. Case Study. A numerical experiment is conducted to demonstrate the effectiveness of the proposed algorithm by comparing centralized method with decentralized method. The control network is composed of $n=5$ agents, which are sequentially connected and numbered as 1 to 5 . To achieve goals, the self-organized agents are able to communicate with one another over a connected, undirected graph. This control network is selected for its clarity and because it is also a problem that requires coordinated behavior among agents. Thus, it is an adequate control network for analysis. In many applications involving networks, nodes can be abstracted as agents that with some capacity make optimal decisions in order to accomplish some designated task. The optimization model can be designed as

$$
\begin{array}{ll}
\min & 5 x_{1}+4 x_{2}+2 x_{3}+13 x_{4}+3 x_{5} \\
\text { s.t. } & -5 \leq x_{1}+x_{2} \leq 5 \\
& -4 \leq x_{2}+x_{3} \leq 5 \\
& -3 \leq x_{1}+2 x_{2} \leq 6 \\
& 1 \leq x_{5} \leq 4 \\
& -2 \leq x_{1} \leq 3 \\
& -4 \leq x_{2} \leq 4 \\
& -6 \leq x_{3} \leq 7 \\
& -7 \leq x_{4} \leq 4 \\
& -5 \leq 2 x_{1}+x_{2}+x_{3} \leq 7 \\
& 0 \leq x_{4}+2 x_{5} \leq 10 \\
& -10 \leq 2 x_{2}+3 x_{3}+x_{4} \leq 10 \\
& -5 \leq x_{3}+x_{4}+x_{5} \leq 9 .
\end{array}
$$

The objective function and the corresponding constraints can be decomposed using the approach mentioned above. The local objective function and corresponding constraints in each node are described in Figure 5.

The evolutionary process of the decision variables can be obtained through the presented algorithm and is illustrated in Figure 6(a). The respective evolutionary process of each local objective function is demonstrated in Figure 6(b).

The detailed numerical results of the decentralized optimization algorithm are shown in Table 1. For comparison, the optimal results obtained by the centralized method are listed in the same table. It can be seen that the decentralized optimization almost converges to the optimal results of centralized optimization. Thus the numerical simulations show the effectiveness of the proposed decentralized algorithm and validate the theoretical analysis. Next, the presented algorithm is examined in practical applications. The present study focuses on two basic operational tasks in HVAC control systems, namely, sensor fault self-repair and device group control.

\section{Sensor Fault Self-Repair Application}

In the decentralized HVAC control system, each sensor is regarded as a low-intelligence node called a sensor-Agent. A sensor-Agent is attached to the CPN, which can be selforganized and self-computed independently. In practice, 


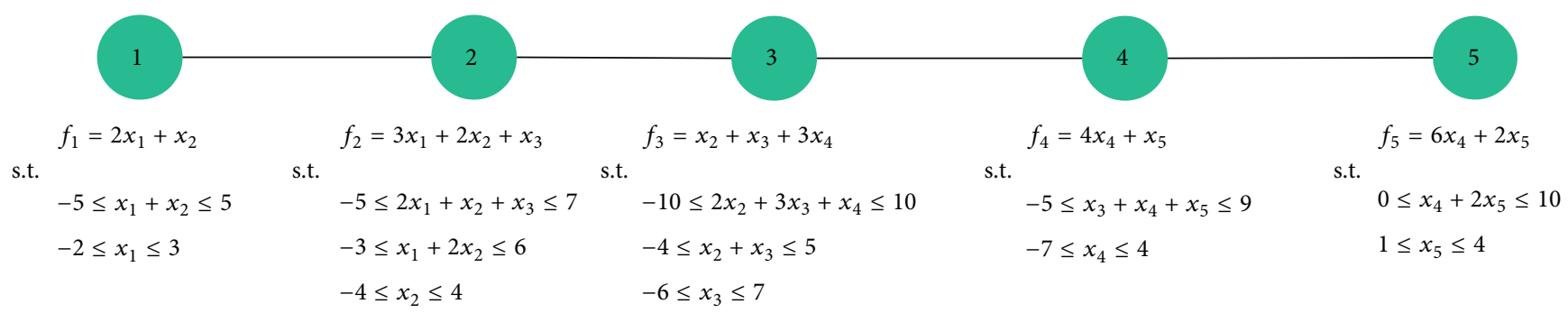

FIGURE 5: The local objective function and corresponding constraints in each node.

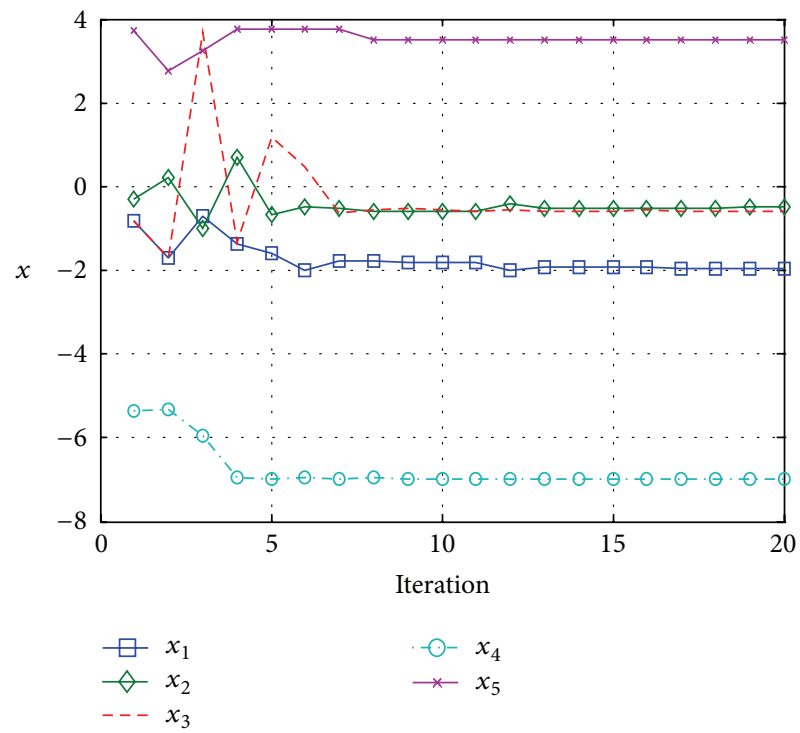

(a)

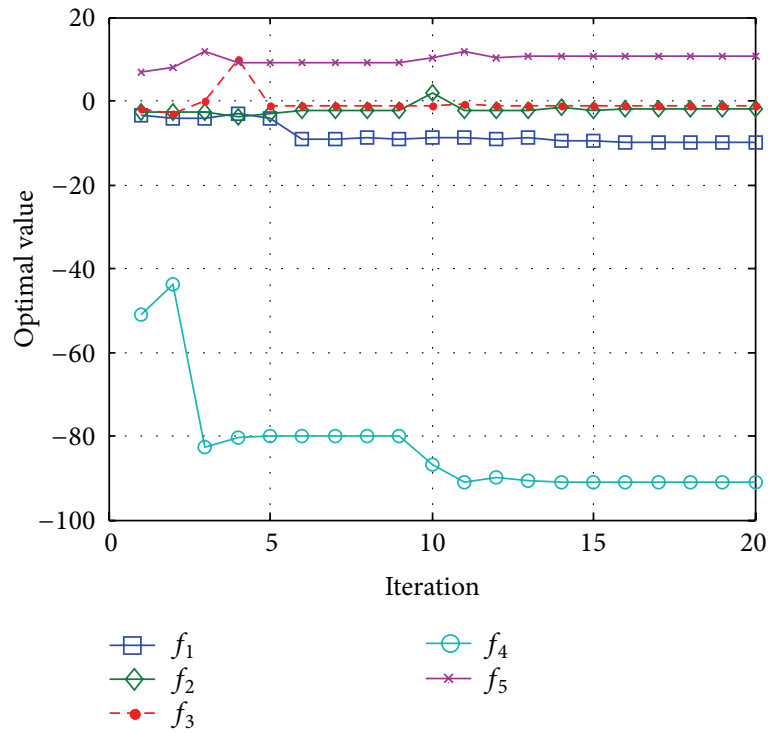

(b)

FIGURE 6: Simulation results of the decentralized optimization. (a) Evolutionary process of decision variables. (b) Evolutionary process of local objectives.

there may be more than one sensor covering a single zone. In this case, the multiple measurements can be combined by averaging or using advanced sensor fusion methods, while the proposed mechanism can still be applied.

4.1. Problem Formulation. Systematic comparison and optimization, a novel method for sensor drift error, is presented in the literature [25] to remedy the deficiencies of the existing SFDD methods in practical engineering. The SFDD in the present study is also formulated as a constrained optimization problem. Due to the fact that faulty sensors are always less in engineered system, the optimization aims to minimize the faulty probability of all the sensors. The equality and inequality constraints are established on the basis of basic physical laws in HVAC system. Thus, the centralized optimization model for sensor fault detection and self-repair can be described as

$$
\begin{aligned}
\min & \sum_{i=1}^{N} f_{i}\left(\left|\mathbf{x}_{i}-\overline{\mathbf{x}}_{i}\right|\right) \\
\text { s.t. } & g_{k}(\mathbf{x}) \leq \mathbf{0}, \quad k=1,2, \ldots, p \\
& h_{j}(\mathbf{x})=\mathbf{0}, \quad j=1,2, \ldots, q,
\end{aligned}
$$

where $\overline{\mathbf{x}}_{i}$ is the measurement of sensor $i$, the optimal solution (true value) of which is $\mathbf{x}_{i}$. Then, $\left|\mathbf{x}_{i}-\overline{\mathbf{x}}_{i}\right|$ can be explained as the measured error of sensor $i$. Parameters $p$ and $q$ denote the quantity of inequality and equality constraints, respectively. The objective function expresses the faulty probability (FP) of sensor $i$ and

$$
f_{i}\left(\left|\mathbf{x}_{i}-\overline{\mathbf{x}}_{i}\right|\right)=1-\exp \left(-\boldsymbol{\alpha} \cdot\left|\mathbf{x}_{i}-\overline{\mathbf{x}}_{i}\right|\right)
$$

where parameter $\boldsymbol{\alpha}>0$ can affect the slope of the faulty probability function. Problem (39) can be transformed into problem (40) on each agent $i, i=1, \ldots, N$, based on the above analysis:

$$
\begin{array}{cl}
\min & f_{i}\left(\left|\mathbf{x}_{i}-\overline{\mathbf{x}}_{i}\right|\right) \\
\text { s.t. } & g_{i}\left(\mathbf{x}_{i}\right) \leq \mathbf{0} \\
& h_{i}\left(\mathbf{x}_{i}\right)=\mathbf{0} .
\end{array}
$$

In the decentralized control system, a flat undirected graph is used to describe the decentralized sensor network. If constraints exist between sensors $i$ and $j$, then these sensors are connected using an undirected edge $\{i, j\}$. The comparison between the centralized and decentralized SFDD methods is 
TABLE 1: Comparison between the centralized and decentralized algorithms.

\begin{tabular}{lcccccc}
\hline Computing method & $x_{1}$ & $x_{2}$ & $x_{3}$ & $x_{4}$ & $x_{5}$ & Objective \\
\hline Centralized & -1.9091 & -0.5455 & -0.6364 & -7.0000 & 3.5000 & -93.500 \\
Decentralized & -1.9115 & -0.5228 & -0.6531 & -6.9950 & 3.4972 & -93.4 \\
Error (\%) & 0.1330 & 4.1577 & 2.6314 & 0.0748 & 0.0740 & 0.1070 \\
\hline
\end{tabular}

TABLE 2: Variables and parameters of the chilled water system.

\begin{tabular}{|c|c|c|c|}
\hline Symbol & Description & Unit & Property \\
\hline$Q_{1}, Q_{2}$ & Supply chilled water flow of Chiller ${ }_{1-2}$ & $\mathrm{~L} \cdot \mathrm{s}^{-1}$ & Parameter \\
\hline$Q_{9}, Q_{10}, Q_{11}, Q_{12}$ & Supply chilled water flow of $\mathrm{FCU}_{1-4}$ & $L \cdot s^{-1}$ & Parameter \\
\hline$Q_{3}$ & Supply chilled water flow of the primary pump side & $\mathrm{L} \cdot \mathrm{s}^{-1}$ & Parameter \\
\hline$Q_{4}$ & Supply chilled water flow of the secondary pump side & $\mathrm{L} \cdot \mathrm{s}^{-1}$ & Parameter \\
\hline$Q_{13}$ & Return chilled water flow of the secondary pump side & $\mathrm{L} \cdot \mathrm{s}^{-1}$ & Parameter \\
\hline$Q_{14}$ & Return chilled water flow of the primary pump side & $\mathrm{L} \cdot \mathrm{s}^{-1}$ & Parameter \\
\hline$T_{1}, T_{2}$ & Supply chilled water temperature of Chiller ${ }_{1-2}$ & ${ }^{\circ} \mathrm{C}$ & Variable \\
\hline$T_{15}, T_{16}$ & Return chilled water temperature of Chiller ${ }_{1-2}$ & ${ }^{\circ} \mathrm{C}$ & Variable \\
\hline$T_{3}$ & Supply chilled water temperature of the primary pump side & ${ }^{\circ} \mathrm{C}$ & Variable \\
\hline$T_{14}$ & Return chilled water temperature of the primary pump side & ${ }^{\circ} \mathrm{C}$ & Variable \\
\hline$T_{4}$ & Supply chilled water temperature of the secondary pump side & ${ }^{\circ} \mathrm{C}$ & Variable \\
\hline$T_{13}$ & Return chilled water temperature of the secondary pump side & ${ }^{\circ} \mathrm{C}$ & Variable \\
\hline$T_{5}, T_{6}, T_{7}, T_{8}$ & Supply chilled water temperature of $\mathrm{FCU}_{1-4}$ & ${ }^{\circ} \mathrm{C}$ & Variable \\
\hline$T_{9}, T_{10}, T_{11}, T_{12}$ & Return chilled water flow of $\mathrm{FCU}_{1-4}$ & ${ }^{\circ} \mathrm{C}$ & Variable \\
\hline
\end{tabular}

illustrated in Figure 7. The presented decentralized algorithm runs in each sensor-Agent and operates at the field level of an HVAC control system.

The chilled water system, which is an essential subsystem in HVAC systems, is used as an example to validate the presented algorithm.

4.2. Chilled Water System Model. In the chilled water system, the bypass valve has a one-way direction. The two parallel chillers of the primary pump side supply chilled water to four parallel Fan-Coil Units (FCUs) through secondary pumps. The return chilled water of each FCU converges to the chillers. The chilled water of the primary and secondary loops is balanced by a bypass pipe between the water distributor and collector. The detailed system structure is illustrated in Figure 8(a). Parameters $Q_{1}-Q_{16}$ are assumed to be given. Variables $T_{1}-T_{16}$ are measured by temperature sensors 1-16. The detailed variables and parameters are listed in Table 2. The constraint model of each sensor-Agent is developed and illustrated in Table 3. The range of the variables can be identified according to the rated values. Supply chilled water temperature sensors $T_{1}-T_{8}$ have a range of $\left[T_{l b}, T_{u b}\right]=$ $[5,10]$, and return chilled water temperature sensors $T_{9}-$ $T_{16}$ have a range of $\left[T_{l b}, T_{u b}\right]=[8,15]$. The decentralized sensor network is established according to their constraint relationship. Figure 8(b) demonstrates the sensor network topology of the chilled water system.
4.3. Simulation Results. The present study assumes that the water flow parameters $\left(\mathrm{L} \cdot \mathrm{s}^{-1}\right)$ are $Q_{1}=60, Q_{2}=90, Q_{3}=150$, $Q_{4}=120, Q_{9}=30, Q_{10}=30, Q_{11}=30, Q_{12}=30, Q_{13}=120$, and $Q_{14}=150$. Parameter $\alpha$ in the objective function is set to 5. When four sensors fail at the same time, the measurements and drift error are set as shown in Figure 9.

The diagnosis results are obtained by implementing the presented algorithm on sensors 1-16. Figure 10 shows the evolutionary process of decision variables. Under mutual negotiation and adjustment, the optimal correction value fluctuates and finally reaches a new equilibrium state. After about 10 iterations, the algorithm becomes convergent. It can be seen that the stable value of faulty sensors has a larger deviation from the measurement than the normal sensors. Table 4 classifies the detailed numerical values to summarize the results clearly.

The FP of each sensor, that is, the objective function of the decentralized optimization, is presented in Figure 11. The FP of the faulty nodes tend to approach 1 . Conversely, the FP of the normal nodes approaches 0 . The results indicate that sensors $3,6,13$, and 15 fail with a probability of 1 , whereas all other sensors are normal. The simulation results presented in the preceding paragraph indicate that the proposed method can realize sensor fault self-repair and adequately solve the problem of drift error. The diagnosis performance of the proposed method depends on the quality and quantity of the constraints involved. For best results, each variable $x_{i}$ 


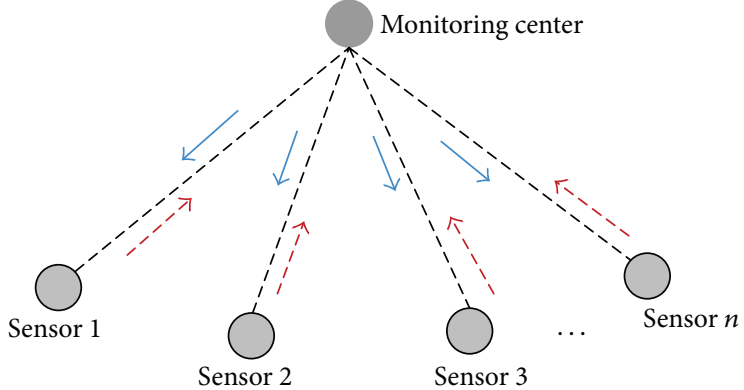

$\longrightarrow$ Master node sends instructions to sensors

$-\rightarrow$ Slave nodes transmit information to master node

(a)

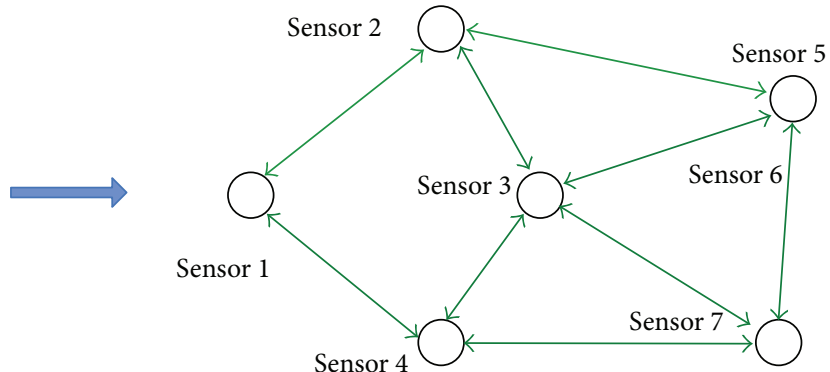

$\leftrightarrow$ Bidirectional information transmission

(b)

FIGURE 7: Comparison between the centralized and decentralized SFDD methods. (a) Conventional centralized diagnostic method. (b) Decentralized diagnostic method.

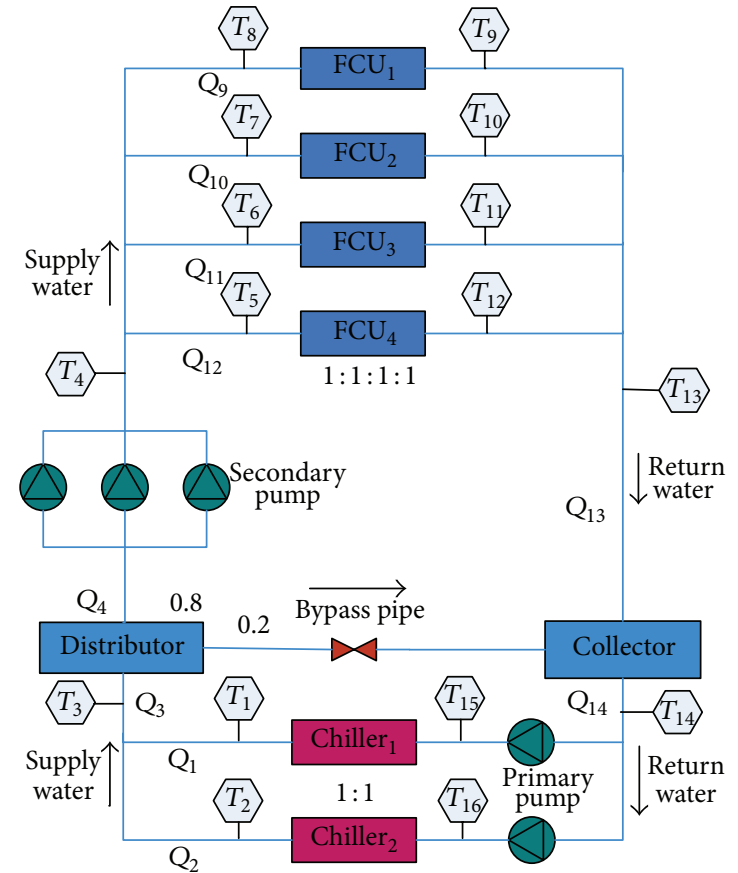

(a)

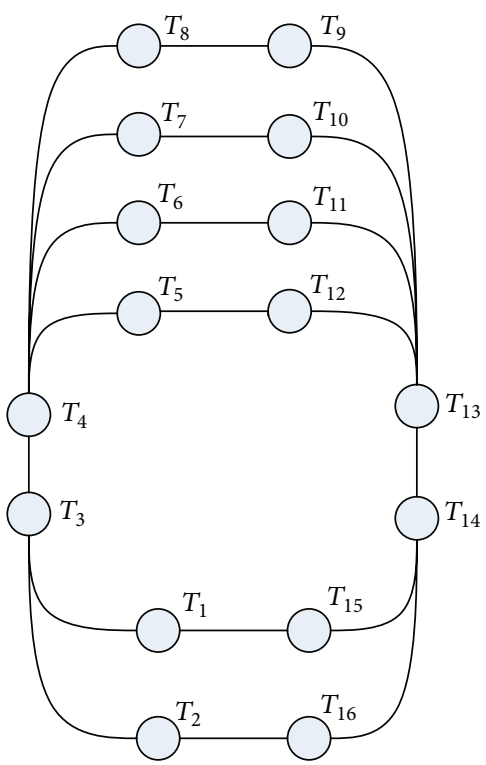

(b)

FIgURE 8: Model of the chilled water system. (a) Detailed system structure. (b) Decentralized sensor network.

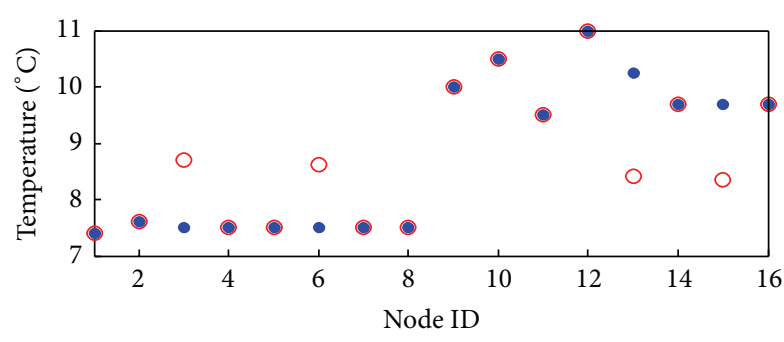

- True

○ Measurement

FIgURE 9: Drift error distribution. has to be involved in at least one equality constraint or two inequality constraints as shown in

$$
\begin{aligned}
& h_{i}\left(x_{i}\right)=h_{j}\left(x_{j}\right), \\
& g_{j}\left(x_{j}\right)>g_{i}\left(x_{i}\right)>g_{k}\left(x_{k}\right),
\end{aligned}
$$

\section{Group Control Application}

An extended application of the presented algorithm is examined in this section. 
Diagnosis result of sensor 1
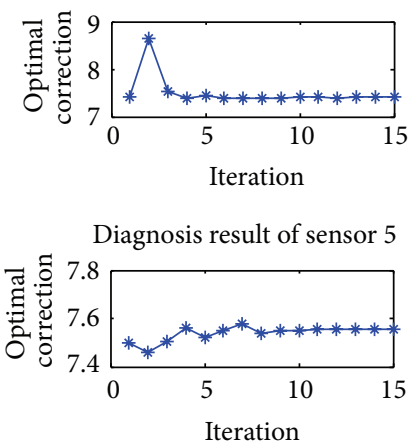

Diagnosis result of sensor 9

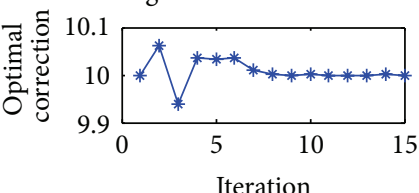

Iteration

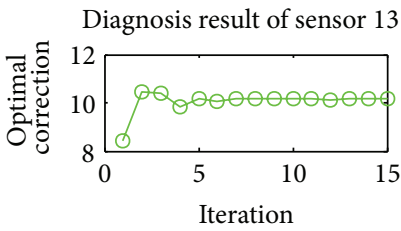

Diagnosis result of sensor 2

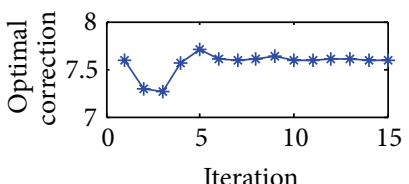

Diagnosis result of sensor 6

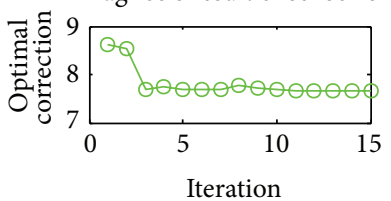

Diagnosis result of sensor 10

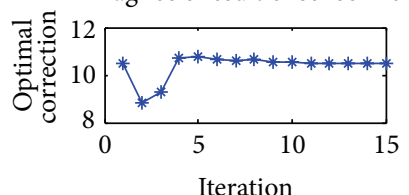

Diagnosis result of sensor 14

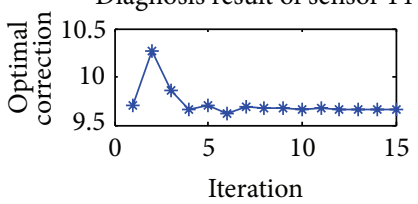

Diagnosis result of sensor 3

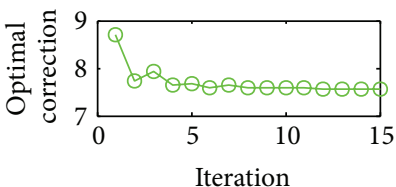

Diagnosis result of sensor 7

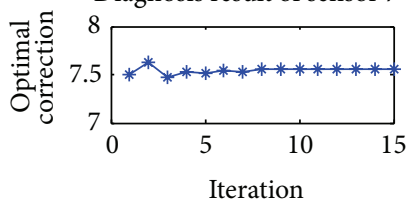

Diagnosis result of sensor 11

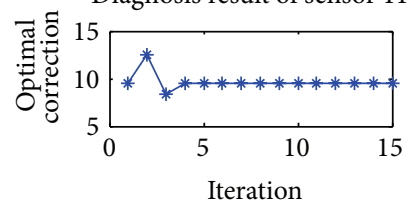

Diagnosis result of sensor 15

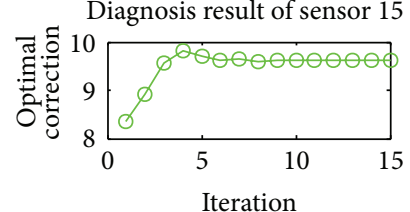

Diagnosis result of sensor 4

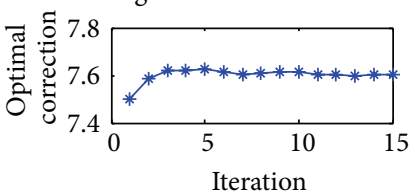

Diagnosis result of sensor 8

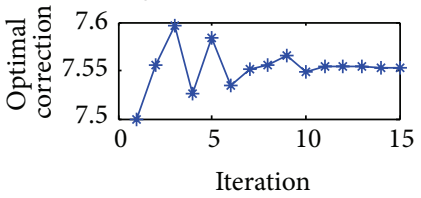

Diagnosis result of sensor 12

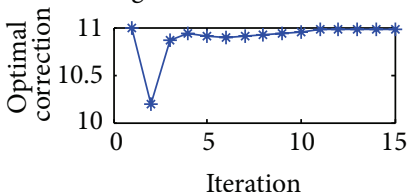

Diagnosis result of sensor 16

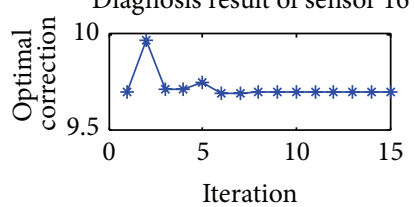

FIGURE 10: Evolutionary process of the decision variables.

TABLE 3: Constraint model of each sensor.

\begin{tabular}{ll}
\hline Sensor ID & Constraint model \\
\hline Sensor 1 & $T_{1}<T_{15}, T_{l b, 1} \leq T_{1} \leq T_{u b, 1}$ \\
Sensor 2 & $T_{2}<T_{16}, T_{l b, 2} \leq T_{2} \leq T_{u b, 2}$ \\
Sensor 3 & $T_{3}<T_{14}, T_{l b, 3} \leq T_{3} \leq T_{u b, 3}, T_{3}=T_{4}$ \\
Sensor 4 & $T_{l b, 4} \leq T_{4} \leq T_{u b, 4}, T_{4}=T_{5}$ \\
Sensor 5 & $T_{5}<T_{12}, T_{l b, 5} \leq T_{5} \leq T_{u b, 5}$ \\
Sensor 6 & $T_{l b, 6} \leq T_{6} \leq T_{u b, 6}, T_{4}=T_{6}$ \\
Sensor 7 & $T_{l b, 7} \leq T_{7} \leq T_{u b, 7}, T_{4}=T_{7}$ \\
Sensor 8 & $T_{l b, 8} \leq T_{8} \leq T_{u b, 8}, T_{4}=T_{8}$ \\
Sensor 9 & $T_{8}<T_{9}, T_{l b, 9} \leq T_{9} \leq T_{u b, 9}$ \\
Sensor 10 & $T_{7}<T_{10}, T_{l b, 10} \leq T_{10} \leq T_{u b, 10}$ \\
Sensor 11 & $T_{l b, 11} \leq T_{11} \leq T_{u b, 11}, T_{6}<T_{11}$ \\
Sensor 12 & $T_{l b, 12} \leq T_{12} \leq T_{u b, 12}$ \\
Sensor 13 & $T_{l b, 13} \leq T_{13} \leq T_{u b, 13}$ \\
& $Q_{9} T_{9}+Q_{10} T_{10}+Q_{11} T_{11}+Q_{12} T_{12}=Q_{13} T_{13}$ \\
Sensor 14 & $T_{l b, 14} \leq T_{14} \leq T_{u b, 14}, T_{14}=T_{15}$ \\
Sensor 15 & $Q_{13} T_{13}+\left(Q_{3}-Q_{4}\right) T_{3}=Q_{14} T_{14}$ \\
Sensor 16 & $T_{l b, 15} \leq T_{15} \leq T_{u b, 15}, T_{16}=T_{15}$
\end{tabular}

5.1. Problem Formulation. The optimal control of devices group plays an important role in HVAC system. The group control can also be abstracted as the constrained optimization model. Taking chillers as an example, the objective function is to minimize the energy consumption of all chillers. The constraints refer to the load balance; that is, the sum of the cooling loads is equal to the demand of the terminal. Thus, the centralized optimization model for the group control can be described as

$$
\begin{array}{ll}
\min & \sum_{i=1}^{N} u_{i} S_{i} \\
\text { s.t. } & \sum_{i=1}^{N} u_{i} p_{i}=L,
\end{array}
$$



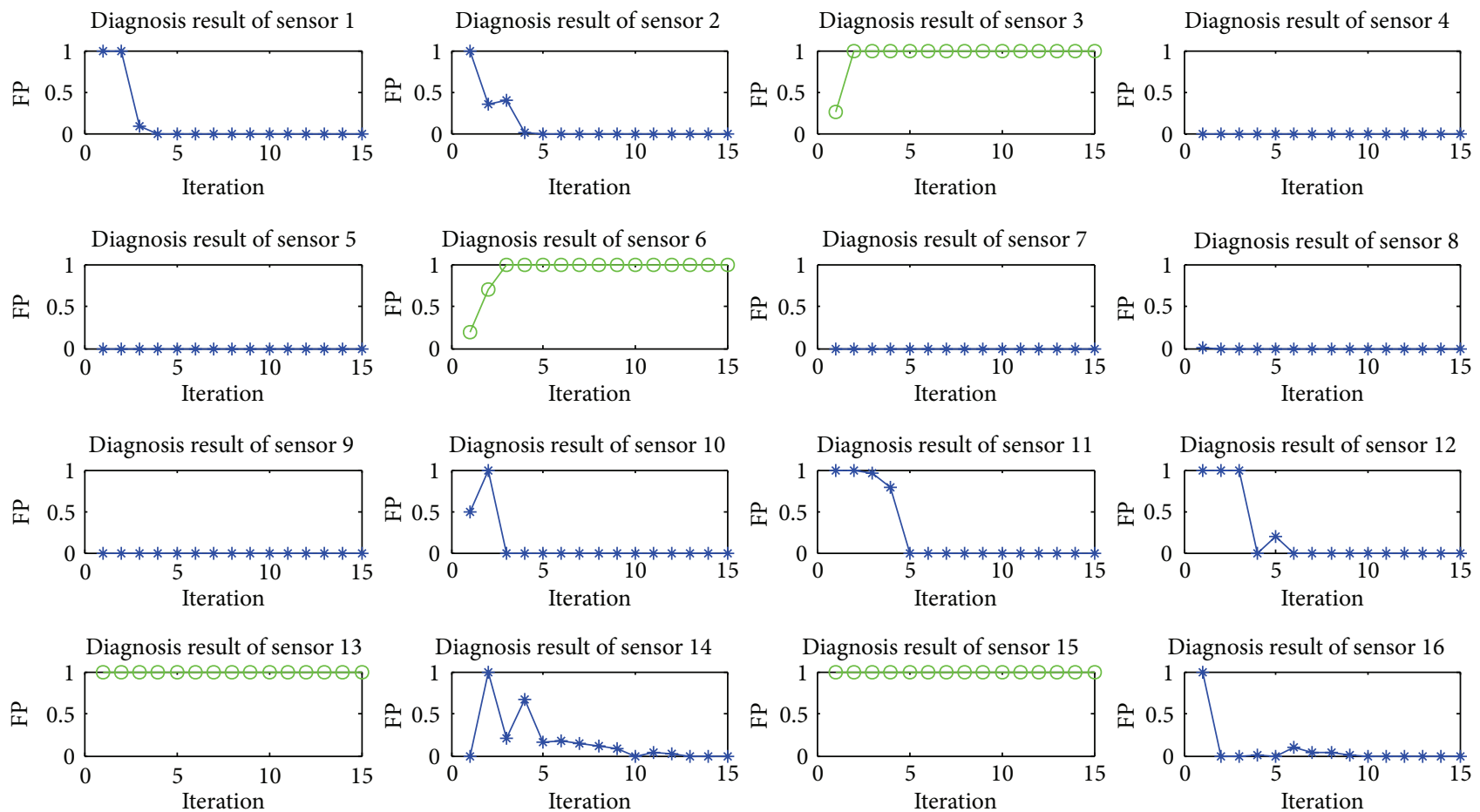

FIGURE 11: Evolutionary process of the faulty probability.

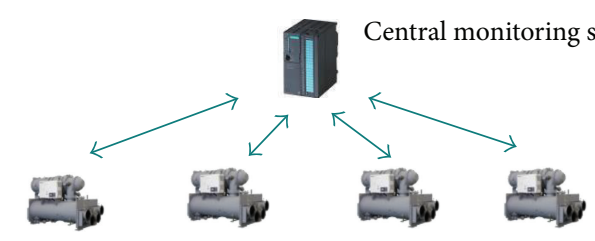

(a)

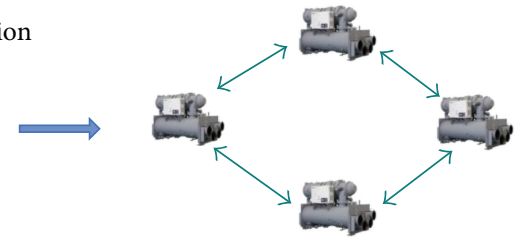

(b)

Figure 12: Comparison between the centralized and decentralized group control. (a) Traditional centralized control method. (b) Decentralized control method.

where $S_{i}$ is the cost of unit $i, N$ is the quantity of devices, $L$ is the total load demand of the terminal, $p_{i}$ is the rated capacity (kW) of chiller $i$, and $u_{i}$ is the status of unit $i$, and

$$
u_{i}= \begin{cases}1, & \text { on } \\ 0, & \text { off. }\end{cases}
$$

Based on the control scheme proposed in the present study, problem (42) can be transformed into problem (44) for agent $i, i=1, \ldots, N$. Consider

$$
\begin{array}{ll}
\min & u_{i} S_{i} \\
\text { s.t. } & \sum_{i=1}^{N} u_{i} p_{i}=L .
\end{array}
$$

The device adopted in the decentralized control system is the updated smart device; that is, each decentralized controller is embedded into the body of chillers and becomes an organic part of each device. Therefore, the presented decentralized control algorithm can be executed in each smart device as demonstrated in Figure 12.

5.2. Simulation Results. The quantity of chillers $n$ is assumed to be 20 . The rated capacity $(\mathrm{kW})$ of chillers $1-20$ is assumed to be $15,20,33,41,57,69,15,12,10,24,12,15,12,69,15,12$, $33,41,57$, and 69 , respectively. To simplify the calculation, the cost $S_{i}, i=1,2, \ldots, n$, of each device is assumed to be identical. The load demand $L=228(\mathrm{~kW})$ is assumed, and the result is expected to be reasonable with a margin error of $0.2(\mathrm{~kW})$. The simulation result is shown in Figure 13 . Chillers 3, 4, 5, 7, 9, 10, 12, and 17 are part of the startup. It can be seen that the summary result satisfies the load demand. The decision process converges at approximately the 300 th iteration, and the consumed time is $35.77 \mathrm{~s}$. However, the result is executed on a common computer with the Von Neumann type. To obtain more precise results, the present study utilizes the decentralized test platform described in the subsequent section. 

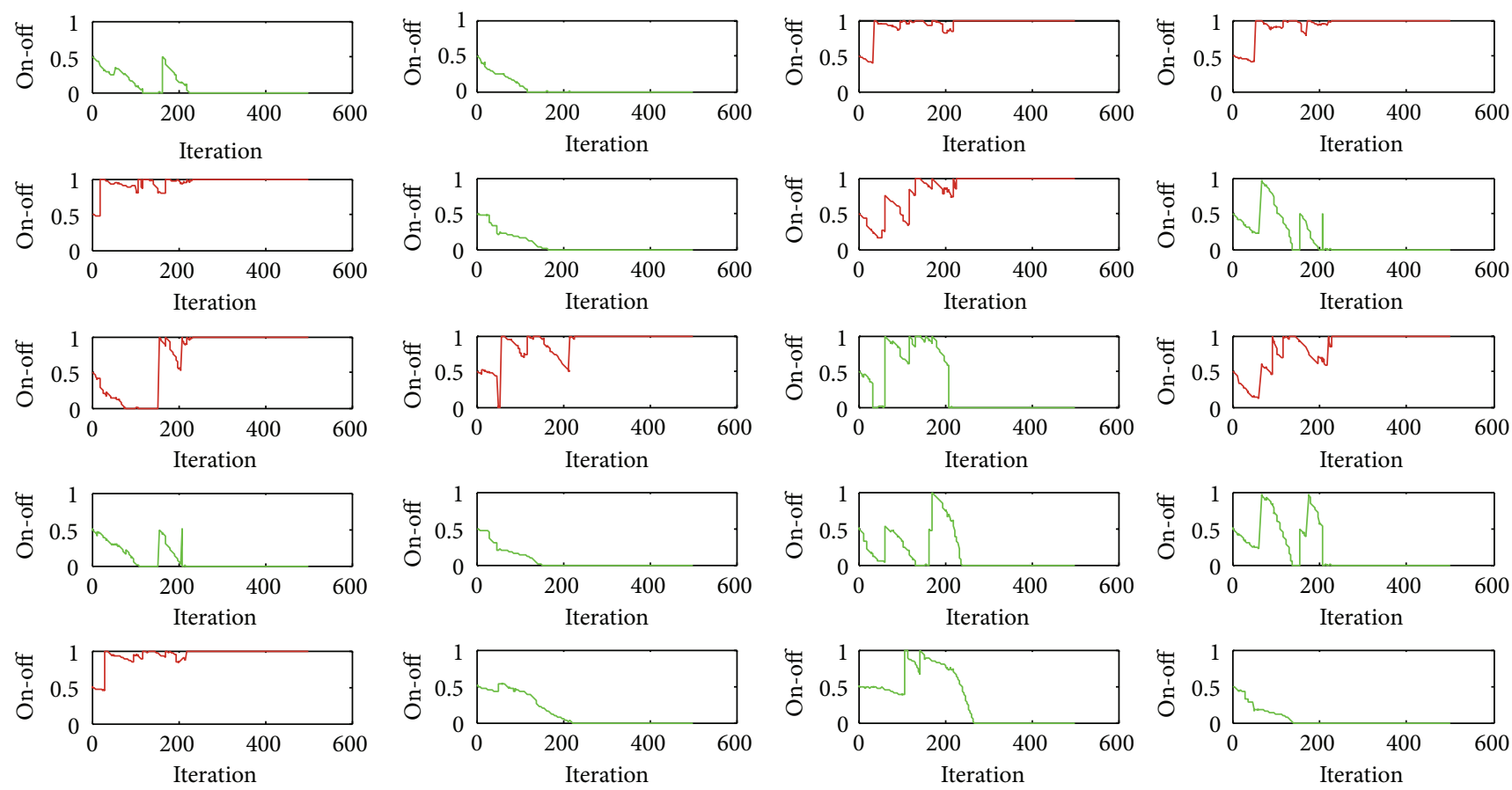

FIGURE 13: Decision process of the group control.

TABLE 4: Numerical results.

\begin{tabular}{|c|c|c|c|c|}
\hline Faulty sensor & Sensor 3 & Sensor 6 & Sensor 13 & Sensor 15 \\
\hline Measurement $\left({ }^{\circ} \mathrm{C}\right)$ & 8.7000 & 8.6250 & 8.4050 & 8.3420 \\
\hline True value $\left({ }^{\circ} \mathrm{C}\right)$ & 7.5000 & 7.5000 & 10.2500 & 9.7000 \\
\hline Repairing value $\left({ }^{\circ} \mathrm{C}\right)$ & 7.4535 & 7.5465 & 10.3200 & 10.1933 \\
\hline
\end{tabular}

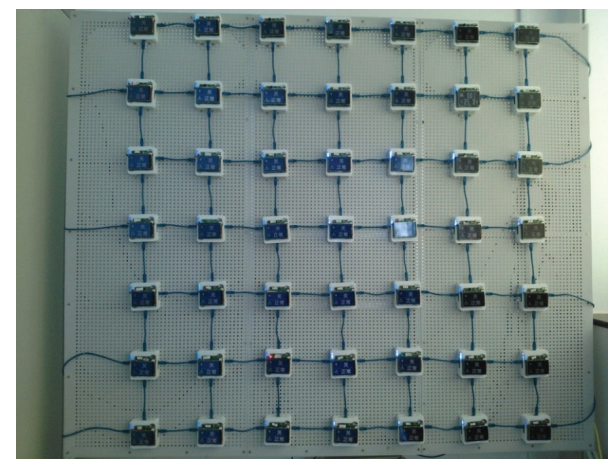

FIGURE 14: Decentralized test platform at Tsinghua University.

\section{Hardware Test}

The decentralized test platform developed in the present study consists of a series of CPNs as shown in Figure 14. In practical engineering, each $\mathrm{CPN}$ is connected to its neighbors through a network cable or wireless communication. In the hardware experiment, SFDD and group control algorithm can be downloaded to each decentralized CPN to run. Each $\mathrm{CPN}$ acts as a sensor-Agent or smart device to test the decentralized algorithm.
The whole system is self-organized and plug-and-play. No configuration or commissioning work is needed. Regardless of the numbers or types of devices within the HVAC systems, a simple connection of neighboring smart devices through RJ45 jacks enables them to communicate with one another and collaboratively work to realize the SFDD and the group control.

\section{Conclusion}

In actual projects, the proprietor has to rely on a system integrator for HVAC control systems. Network construction is a complex and time-consuming process because a considerable amount of secondary development, such as configuration and commissioning, is necessary. In the key procedure, the control algorithm and model are written into the centralized controller, which needs to be reprogrammed from case to case because the system configuration or device type changes. Therefore, the development and operation involved in this undertaking incur high maintenance and labor costs.

A novel idea for the decentralized control method is proposed to solve actual engineering problems. With the rapid development of the electronic industry, smart hardware has been widely employed in different fields. According to the vision of a decentralized method, traditional devices can be 
upgraded and transformed into smart devices through the incorporation of a decentralized control chip. The accurate model and decentralized algorithm can be written into this chip. Smart devices communicate with their neighboring nodes and work collaboratively to perform the control tasks. In this case, complicated onsite modeling, configuration, commissioning, and other developing work can be simplified to the wiring of communication connection among smart devices, which can easily adapt to any change in subsystems and can be plug-and-play. Therefore, most tasks can be completed on the field level of the HVAC control system.

The proposed method meets the requirements of the modern HVAC control system and is adaptive to other largescale distributed control systems. However, the present study is an elementary exploration into the decentralized control system and needs further investigation.

\section{Competing Interests}

The authors declare that there is no conflict of interests regarding the publication of this paper.

\section{Acknowledgments}

The authors would like to acknowledge the support provided by Tsinghua University-UTC Research Center for Integrated Building Energy, Safety and Control Systems.

\section{References}

[1] V. Reppa, P. Papadopoulos, M. M. Polycarpou, and C. G. Panayiotou, "A distributed architecture for HVAC sensor fault detection and isolation," IEEE Transactions on Control Systems Technology, vol. 23, no. 4, pp. 1323-1337, 2015.

[2] S. M. Namburu, M. S. Azam, J. Luo, K. Choi, and K. R. Pattipati, "Data-driven modeling, fault diagnosis and optimal sensor selection for HVAC chillers," IEEE Transactions on Automation Science \& Engineering, vol. 4, no. 3, pp. 469-473, 2007.

[3] S. Wang and J. Qin, "Sensor fault detection and validation of VAV terminals in air conditioning systems," Energy Conversion \& Management, vol. 46, no. 15-16, pp. 2482-2500, 2005.

[4] W.-Y. Lee, J. M. House, and N.-H. Kyong, "Subsystem level fault diagnosis of a building's air-handling unit using general regression neural networks," Applied Energy, vol. 77, no. 2, pp. 153-170, 2004.

[5] L. Paradis and Q. Han, "A survey of fault management in wireless sensor networks," Journal of Network \& Systems Management, vol. 15, no. 2, pp. 171-190, 2007.

[6] K. Cohen and A. Leshem, "Energy-efficient detection in wireless sensor networks using likelihood ratio and channel state information," IEEE Journal on Selected Areas in Communications, vol. 29, no. 8, pp. 1671-1683, 2011.

[7] W. Liu, S. Zhang, and J. Fan, "A diagnosis-based clustering and multipath routing protocol for wireless sensor networks," International Journal of Distributed Sensor Networks, vol. 2012, Article ID 504205, 11 pages, 2012.

[8] H. Han, B. Gu, T. Wang, and Z. R. Li, "Important sensors for chiller fault detection and diagnosis (FDD) from the perspective of feature selection and machine learning," International Journal of Refrigeration, vol. 34, no. 2, pp. 586-599, 2011.
[9] X. Luo, M. Dong, and Y. Huang, "On distributed fault-tolerant detection in wireless sensor networks," IEEE Transactions on Computers, vol. 55, no. 1, pp. 58-70, 2006.

[10] B. Krishnamachari and S. Iyengar, "Distributed Bayesian algorithms for fault-tolerant event region detection in wireless sensor networks," IEEE Transactions on Computers, vol. 53, no. 3, pp. 241-250, 2004.

[11] B. Fan, Z. Du, X. Jin, X. Yang, and Y. Guo, "A hybrid FDD strategy for local system of AHU based on artificial neural network and wavelet analysis," Building \& Environment, vol. 45, no. 12, pp. 2698-2708, 2010.

[12] X. Xu, F. Xiao, and S. Wang, "Enhanced chiller sensor fault detection, diagnosis and estimation using wavelet analysis and principal component analysis methods," Applied Thermal Engineering, vol. 28, no. 2-3, pp. 226-237, 2008.

[13] Z. Du and X. Jin, "Detection and diagnosis for sensor fault in HVAC systems," Energy Conversion \& Management, vol. 48, no. 3, pp. 693-702, 2007.

[14] T. I. Salsbury and R. C. Diamond, "Fault detection in HVAC systems using model-based feedforward control," Energy \& Buildings, vol. 33, no. 4, pp. 403-415, 2001.

[15] Z. Hou, Z. Lian, Y. Yao, and X. Yuan, "Data mining based sensor fault diagnosis and validation for building air conditioning system," Energy Conversion \& Management, vol. 47, no. 15-16, pp. 2479-2490, 2006.

[16] S. Wang, Q. Zhou, and F. Xiao, "A system-level fault detection and diagnosis strategy for HVAC systems involving sensor faults," Energy \& Buildings, vol. 42, no. 4, pp. 477-490, 2010.

[17] H. Wang, T.-Y. Chai, J.-L. Ding, and M. Brown, "Data driven fault diagnosis and fault tolerant control: some advances and possible new directions," Acta Automatica Sinica, vol. 35, no. 6, pp. 739-747, 2009.

[18] X.-G. Yan and C. Edwards, "Fault estimation for single output nonlinear systems using an adaptive sliding mode estimator," IET Control Theory \& Applications, vol. 2, no. 10, pp. 841-850, 2008.

[19] X. Zhang, T. Parisini, and M. Polycarpou, "Sensor bias fault isolation in a class of nonlinear systems," IEEE Transactions on Automatic Control, vol. 50, no. 3, pp. 370-376, 2005.

[20] T. Guo, "Decentralized control for large-scale interconnected nonlinear systems based on barrier Lyapunov function," Mathematical Problems in Engineering, vol. 2015, Article ID 494721, 6 pages, 2015.

[21] Q. Shen, Study on Architecture of Decentralized System in Intelligent Building, Tsinghua University, Beijing, China, 2015.

[22] M. V. Afonso, J. M. Bioucas-Dias, and M. A. Figueiredo, "Fast image recovery using variable splitting and constrained optimization," IEEE Transactions on Image Processing, vol. 19, no. 9, pp. 2345-2356, 2010.

[23] G. Inalhan, D. M. Stipanovic, and C. J. Tomlin, "Decentralized optimization, with application to multiple aircraft coordination," in Proceedings of the 41st IEEE Conference on Decision and Control, vol. 1, pp. 1147-1155, Las Vegas, Nev, USA, December 2003.

[24] R. Rastegar, "On the optimal convergence probability of univariate estimation of distribution algorithms," Evolutionary Computation, vol. 19, no. 2, pp. 225-248, 2011.

[25] Y. Jiang and W. Zhu, "Sensor fault detection in heating, ventilation and air-conditioning systems," Journal of Tsinghua University, vol. 39, no. 12, pp. 54-61, 1999 (Chinese). 


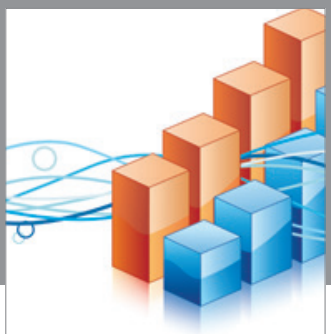

Advances in

Operations Research

vatem alat4

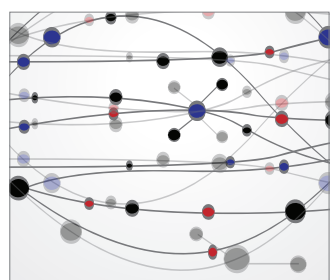

\section{The Scientific} World Journal
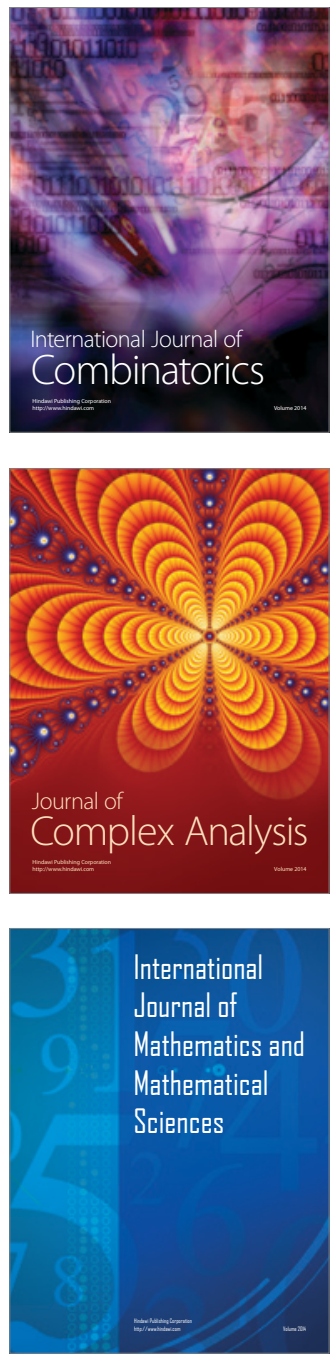
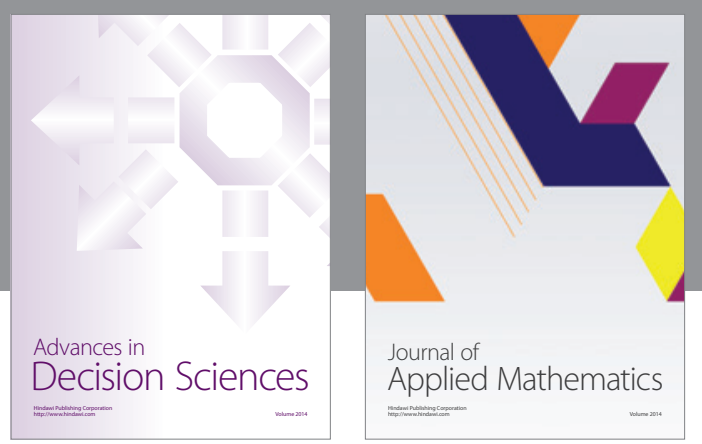

Algebra

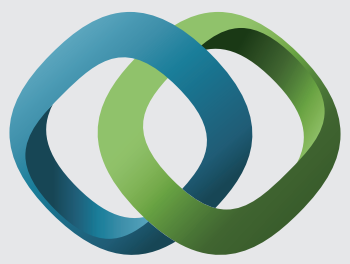

\section{Hindawi}

Submit your manuscripts at

http://www.hindawi.com
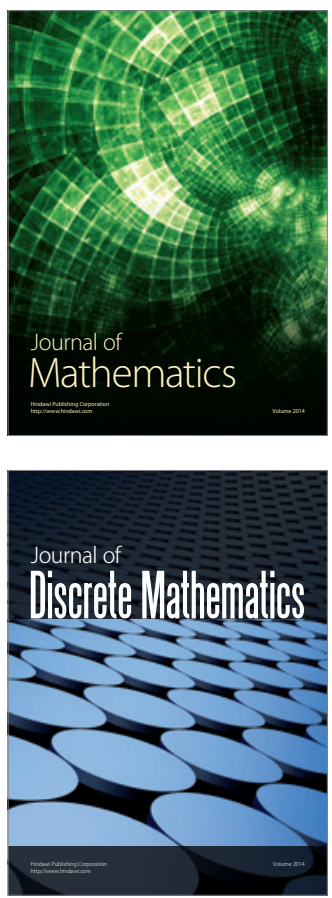

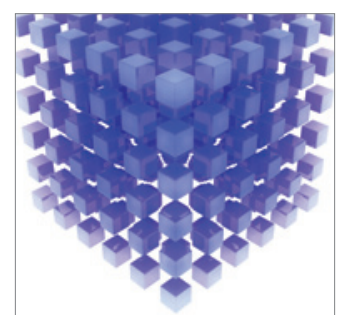

Mathematical Problems in Engineering
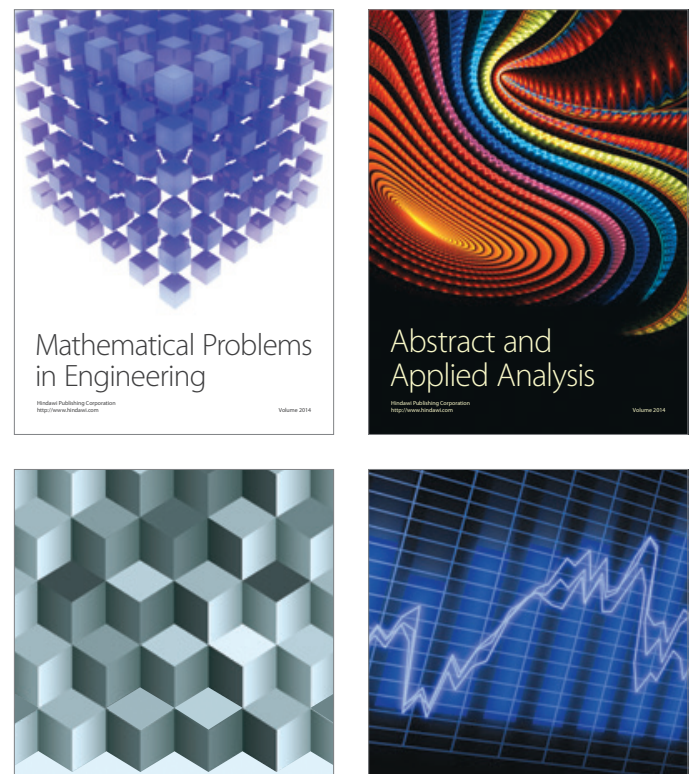

Journal of

Function Spaces

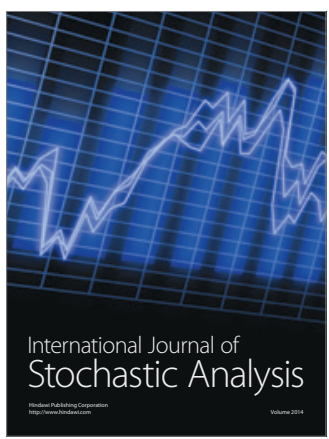

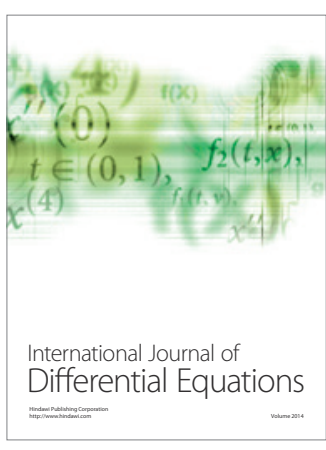
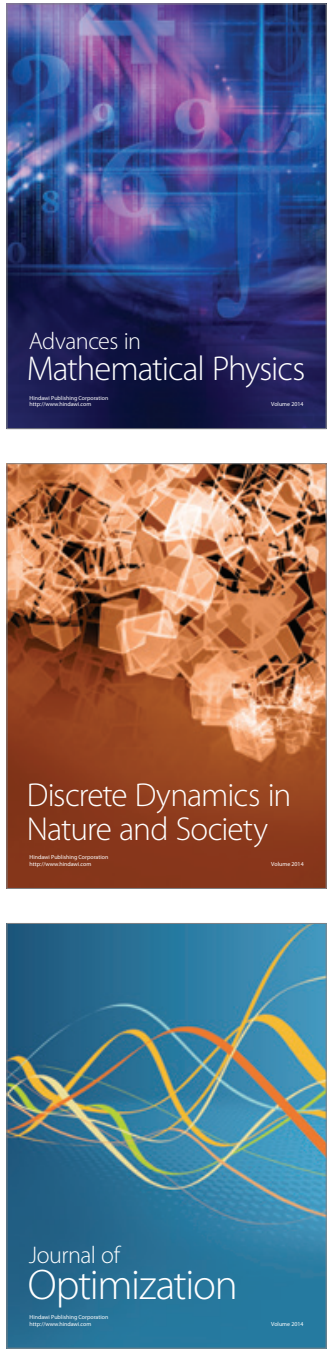